\title{
Development of Sound Insulation Panel From Oil Palm Fiber
}

\author{
Kittichat Homaswin*, Natawut Chaiwino \\ Department of Technical Education \\ Rajamangala University of Technology Thanyaburi \\ Pathum Thani, Thailand \\ *kittichat_h@rmutt.ac.th
}

\begin{abstract}
The purpose of this research is to develop sound insulation panel from oil palm fiber (OPF). The study compared panels made from OPF with other acoustic panels in the market. This raw material is a byproduct from agricultural harvest. Hot press technique with the pressure of $400 \mathrm{~kg} / \mathrm{m3}$ was used to create the panel with the thickness of $20 \mathrm{~mm}$. The temperature during the compression was $150^{\circ} \mathrm{C}$, and the force used for pressing the panels for 8 minutes ranged between $25-30 \mathrm{~kg} / \mathrm{m} 2$. The amount of pMDI applied as a binding agent was $5 \%$ by weight of the panel. Sound absorption tests were carried out in a test box with sound level meters. The results of the tests showed that the sound-absorbing property of the developed OPF panels with holed surface is greater than that of the traditional OPF panels and acoustic boards. It was found that different sizes and patterns of holes have an effect on sound-absorbing capacity. The developed OPF panels showed a good sound-absorbing capability in medium-to-high frequency $(500 \mathrm{~Hz}-2000 \mathrm{~Hz})$. The best sound insulation panel made from OPF is the panel with a $5 \%$ by weight of pMDI and has a density of $400 \mathrm{~kg} / \mathrm{m} 3$. The physical and mechanical properties of the panels comply with the standard of strength and mechanical properties of soft boards (Library of Congress Cataloging - In Publication Data).
\end{abstract}

Keywords—oil palm fiber, sound insulation, hot pressing process

\section{INTRODUCTION}

The noise pollution is a serious problem and trends to increase due to rapid urbanization. The competition in business area and housing development has significantly affected the environment and created pollution. One of the most obvious examples in Thailand is the residential community or educational institutions located around Suvarnabhumi airport which experiences severe noise pollution leading to health and wellness issues.

The architectural design to reduce the noise pollution using sound-absorbing insulation such as acoustic foam sheets and glass fiber insulation has been widespread to control and prevent noise caused by the source inside the building. However, these sound-absorbing insulation is made from synthetic materials which is often concerned with health safety of users. In addition, many materials used in a production process must be imported from abroad [1]. Therefore, many natural materials have been used instead. Such natural materials also reduce energy consumption, chemicals, and toxins created in the production process of synthetic materials [2].

Oil palm is one of the cash crops in Thailand with a yield of 3.256 million tons per year, and the amount of oil palm fiber (OPF) remaining from agriculture is about 64,000 tons per year [1]. The remaining OPF have been processed into the agricultural industry but some are still not utilized [1]. OPF can also be used as an extra component to create an insulation sheet to absorb sound in the building [3]. The use of OPF insulation panel is considered as another way to reduce the volume and increase the value of agricultural waste. Therefore, this study aims to improve the efficiency of sound absorption and develop the insulation panel made from OPF into a commercial product.

\section{LITERATURE REVIEW}

Hearing is one of the most important communications for humans. It may be inferior to seeing with eyes. However, eyes can be closed when facing an excessive amount of light or unpleasant images. Ears must be listen to both satisfying and unsatisfying sound. To prevent noise, therefore, it is necessary to start preventing from an environment before noise reaches the ears. Sound control in the building [4] is used to improve the hearing condition and control unnecessary noise. Sound in the building consists of 2 types: direct noise and reverberant noise.

While direct Noise can be reduced by a barrier between the sound source and the listener, reverberant noise can be reduced by sound absorbing materials on the walls, especially the side of walls that causes a lot of resonance.

Undesirable sounds may be decreased by reducing the resonance with materials which has a better capable of sound absorption than does glass, plaster, or concrete. Each type of materials has different qualities of sound absorption according to the frequency of sound. Sound absorbing materials can be categorized into 4 types as follows:

- Porous type is transparent, light, sponge-like, and suitable for high frequency sounds

- Membrane type is suitable for low frequency sounds

- Resonance-absorbing type can adjust the size to suit the frequency range of the sound, and can absorb the narrow wave sound 
- Perforated type is suitable for medium frequency sound and capable of adjusting the shape as well as the distance between two sheets to suit the frequency of the sound

\section{A. Properties of oil palm fiber}

Oil palm fiber has an average length of about 5 to 7 centimeters, a low curvature, and high resistance to humidity and microbial destruction. It is flexible, durable, long-lasting, and environmentally friendly. Therefore, it has been used for industrial purposes as biomass fuels [5]. The experimental study on natural fibers shows that a coconut fiber can be a great soundabsorbing material and heat insulation [6].

The insulation panel from OPF with a density of 400 $\mathrm{kg} / \mathrm{m} 3$, a thickness of $20 \mathrm{~mm}$, and $5 \%$ glue has physical and mechanical properties which meet the standard of Strength and Mechanical Properties of Soft Boards (Library of Congress Cataloging - In - Publication). The panel can absorb sound at a maximum of 29.42 percent.

\section{RESEARCH OBJECTIVES}

- To develop sound-absorbing panels made from OPF

- To study the properties of OPF panels and develop them to meet the standard of Soft Boards

- To increase the sound-absorbing efficiency of OPF panels

- To compare sound-absorbing properties of OPF panel with the properties of those available in the market (as compared)

\section{METHOD}

The research titled development of sound insulation panel from OPF is an experimental research divided into three phases; panel development, physical and mechanical property testing, and sound absorption testing.

A total of forty OPF panels were formed with the size of 30 $\mathrm{cm} \times 30 \mathrm{~cm}$, the thickness of $20 \mathrm{~mm}$, and the density of 400 $\mathrm{kg} / \mathrm{m} 3$. The requirements of the low density panel (soft board) with a density of about $200-400 \mathrm{~kg} / \mathrm{m} 3$ were adopted. Five percent glue was employed with an amount of oil palm fibers. The calculated amount of oil palm fibers were put into a glue mixer, and glue was consistently sprayed over oil palm fibers as shown in Fig. 1. Oil palm fibers were put into a mold with a size of $30 \mathrm{~cm} \times 30 \mathrm{~cm}$ (wide $\mathrm{x}$ long) as illustrated in Fig. 2. A panel was compressed by compression machine until the specified thickness was achieved (Fig. 3 and 4).

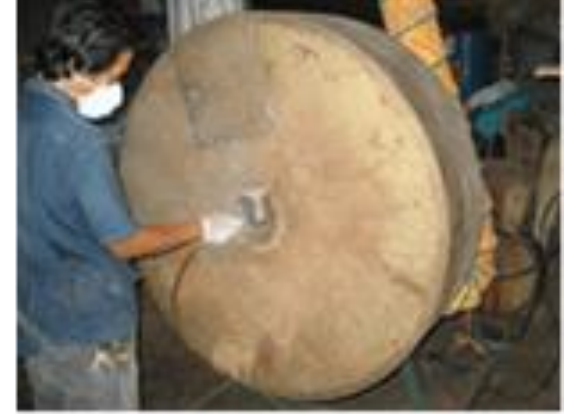

Fig. 1. Glue spraying in a glue mixer.

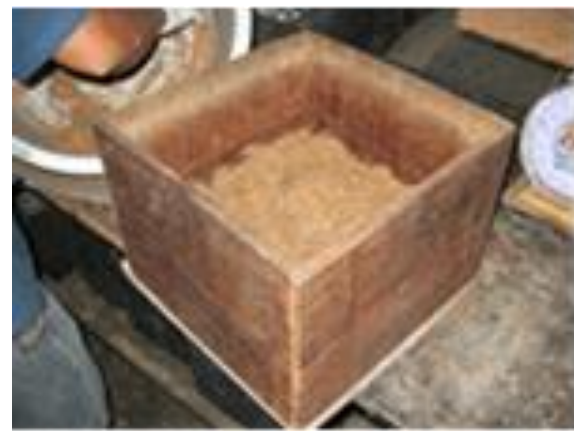

Fig. 2. Putting oil palm fibers into a mold.

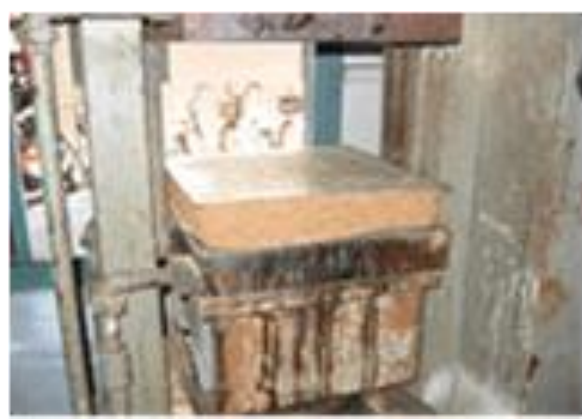

Fig. 3. Compression machine.

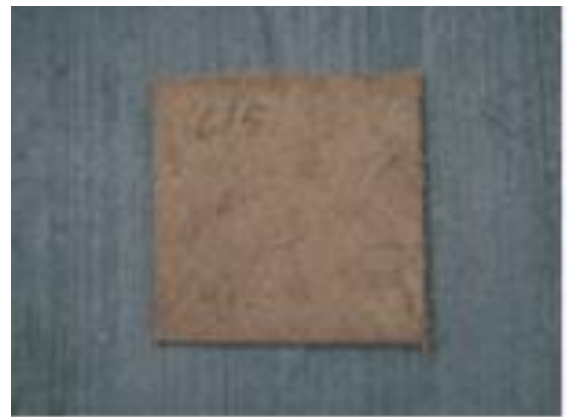

Fig. 4. OPF panel

Tests were done to obtain physical and mechanical properties according to the standard of low density panels (soft board) as shown in Table 1. The required properties consist of density, specific gravity, modulus of elasticity, modulus of 
rupture, tensile strength, and water absorption. The specimens were trimmed to size and tested to find physical and mechanical properties. Two samples were used for each test

\section{A. Physical properties testing}

1) Density: D: Samples with the size of $100 \mathrm{~mm} \times 100 \mathrm{~mm}$ were determined for the density test. Samples were weighed, and their thicknesses measured at $25 \mathrm{~mm}$ away from four edges were averaged. The density was calculated using the equation:

$$
\text { Density }=\mathrm{m} / \mathrm{v}
$$

where: $\mathrm{m}=$ weight $(\mathrm{g})$

$$
\mathrm{v}=\text { volume }(\mathrm{cm} 3)
$$

2) Water absorption by volume: WA: Samples with the size of $50 \mathrm{~mm} \times 50 \mathrm{~mm}$ were weighed and then were immersed in water at room temperature for 24 hours. Samples were separated and set in the direction perpendicular to the water surface, allowing the top edge to be below the surface level about $25 \mathrm{~mm}$. Water absorption by volume can be determined from sample weights before and after immersion in water, and the equations are expressed as:

$$
\text { Water absorption }(\%)=[(\mathrm{v} 2-\mathrm{v} 1) / \mathrm{v} 1] \times 100
$$

where: $\mathrm{v} 1$ = weight before immersion $(\mathrm{g})$

$$
\mathrm{v} 2=\text { weight after immersion }(\mathrm{g})
$$

TABLE I. STANDARD OF LOW DENSITY PANELS (SOFT BOARD) STRENGTH AND MECHANICAL PROPERTIES OF SOFT BoARDS [6]

\begin{tabular}{|l|l|l|}
\hline \multicolumn{1}{|c|}{ Property } & \multicolumn{1}{|c|}{ Unit } & \multicolumn{1}{|c|}{$\begin{array}{c}\text { Value for structural } \\
\text { Insulation board }\end{array}$} \\
\hline Density & $\mathrm{g} / \mathrm{cm} 3$ & $0.14-0.43$ \\
\hline Specific gravity & - & $0.16-0.42$ \\
\hline $\begin{array}{l}\text { Modulus of elasticity } \\
\text { (bending) }\end{array}$ & $\mathrm{MPa}$ & $172.37-861.85$ \\
\hline Modulus of rupture & $\mathrm{MPa}$ & $1.379-5.52$ \\
\hline $\begin{array}{l}\text { Tensile strength perpendicular } \\
\text { to surface }\end{array}$ & $\mathrm{MPa}$ & $0.16-0.4$ \\
\hline $24-$ hour water absorption & $\%$ by volume & $1-10$ \\
\hline
\end{tabular}

\section{B. Mechanical properties testin}

1) Modulus of rupture (MOR) and Modulus of elasticity (MOE): Samples were tested by being placed on the supports whose spacing was 16 times of the specified thickness of the samples, allowing both ends of samples to overhang the supports $12.5 \mathrm{~mm}$. Concentrated load was applied at the middle of the samples at the constant rate of approximately 10 $\mathrm{mm} / \mathrm{second}$. The test must last more than 30 seconds but less than 120 seconds. Modulus of rupture and modulus of elasticity can be calculated according to the following equations:

$$
\mathrm{MOR}=3 \mathrm{WI} \lambda / 2 \mathrm{bd} 2
$$

where: $\mathrm{MOR}=$ modulus of rupture $(\mathrm{Mpa})$

$$
\begin{aligned}
& \mathrm{W}=\text { failure load }(\mathrm{N}) \\
& \mathrm{I} \lambda=\text { support spacing }(\mathrm{mm})
\end{aligned}
$$

$$
\begin{aligned}
& \mathrm{b}=\text { sample width }(\mathrm{mm}) \\
& \mathrm{d}=\text { average sample thickness }(\mathrm{mm})
\end{aligned}
$$$$
\text { MOE } \Delta=\Delta \mathrm{WI} \lambda / 4 \mathrm{bd} 3 \Delta \mathrm{S}
$$

where: $\mathrm{MOE}=$ modulus of elasticity $(\mathrm{Mpa})$

$\Delta \mathrm{W}=$ increased load in linear portion of the curve

(N)

$$
\begin{aligned}
\mathrm{I} \lambda & =\text { support spacing }(\mathrm{mm}) \\
\mathrm{b} & =\text { sample width }(\mathrm{mm}) \\
\mathrm{d} & =\text { average sample thickness }(\mathrm{mm}) \\
\Delta \mathrm{S}= & \text { increased deflection in linear portion of the } \\
& \quad \text { curve }(\mathrm{mm})
\end{aligned}
$$

2) Tensile strength perpendicular to the surface: Both sample surfaces were tested by being attached to the pull plate using synthetic adhesive resins of which the adhesive forces are greater than those within samples. Samples were pulled apart, generally separated in the filling layers, by a pulling machine. Increased rate of pulling force was kept constant, and the test must last more than 30 seconds but less than 120 seconds. Tensile strength perpendicular to the surface can be defined as:

Tensile strength perpendicular to the surface $=$ ultimate tensile force $(\mathrm{N})$ / sample width $(\mathrm{mm}) \mathrm{x}$ sample length $(\mathrm{mm})$

3) Surface design of OPF panel: Six patterns of panel surfaces designed from three different size circles (equal total area) were plotted on plotter papers by AutoCAD software. The papers were attached onto the OPF panels, and then the panels were drilled to make holes of specified diameters. The details of each pattern are depicted from Fig. 5 to Fig. 10.

4) Sound absorption: OPF panels with six design patterns were tested for sound absorption and compared to the soundabsorbing insulations available in the market.
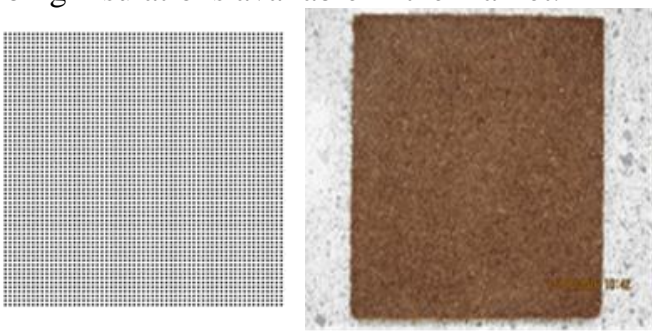

Fig. 5. Pattern 1: 1-mm-diameter circle shaped spot. 


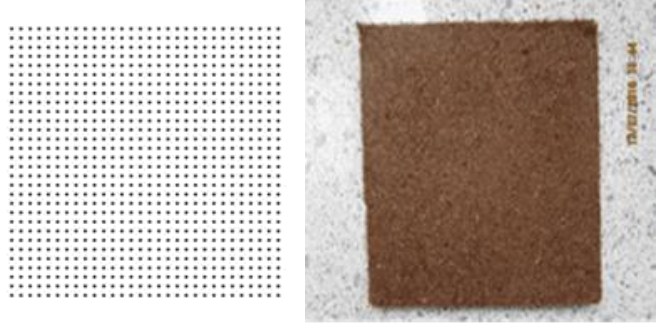

Fig. 6. Pattern 2: 2-mm-diameter circle shaped spot

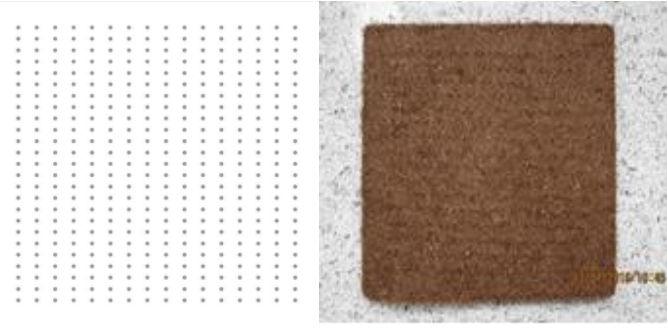

Fig. 7. Pattern 3: 3-mm-diameter circle shaped spot

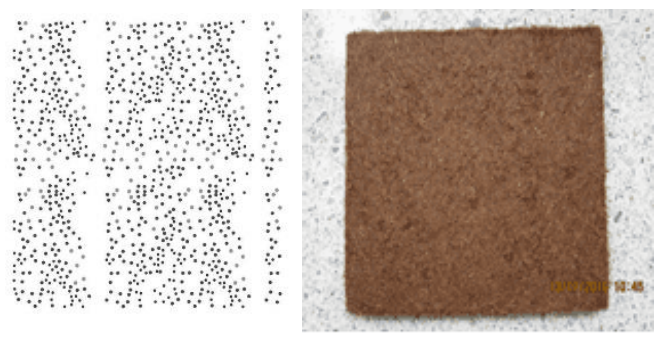

Fig. 8. Pattern 4: Mixed circle shaped spot of 1-mm, 2-mm, and 3-mm diameter

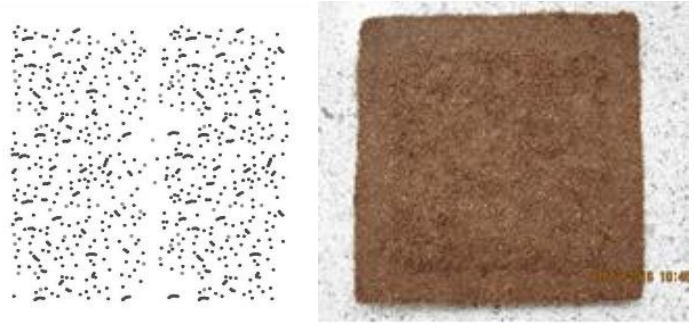

Fig. 9. Pattern 5: Mixed circle shaped spot of 1-mm, 2-mm, and 3-mm diameter

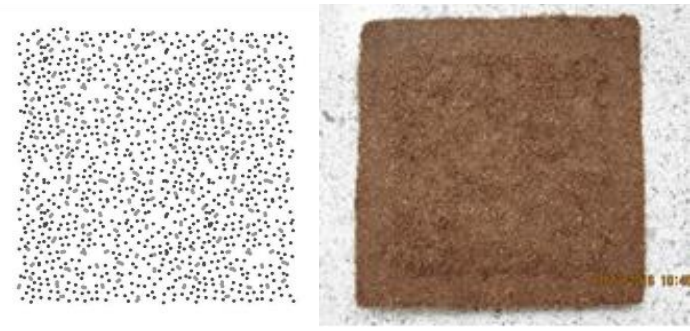

Fig. 10. Pattern 6: Mixed circle shaped spot of 1-mm, 2-mm, and 3-mm diameter
The types of panels with different densities and thicknesses used in the sound absorption tests are summarized in Table 2.

\section{Working principle of a sound test box}

By using the principle of sound reflection, the soundabsorbing efficiency of the panels can be determined. Sound wave created from a sound source will reflect off at the same angle as it hit a testing panel [4]. As a result, a receiver set in the same angle will collect a reflected sound wave, and a decrease in value is an amount absorbed by the panels. Three significant factors in comparison of sound-absorbing or sound-reflecting efficiency of a material must be considered as follows:

- The frequency and volume level of sound must be constant during the test

- The distance between the end of the sound pipe and the microphone must be constant

- The angle of incident must be constant during the test

TABLE II. PROPERTIES OF SOUND-ABSORBING PANELS

\begin{tabular}{|l|l|l|l|}
\hline $\begin{array}{c}\text { Panel } \\
\text { No. }\end{array}$ & \multicolumn{1}{|c|}{ Type of panels } & \multicolumn{1}{|c|}{$\begin{array}{c}\text { Density } \\
\text { kg/m3( }\end{array}$} & \multicolumn{1}{|c|}{$\begin{array}{c}\text { Thickness } \\
(\mathbf{m m})\end{array}$} \\
\hline- & No panel & - & - \\
\hline A1 & Acoustic board 1 & 390 & 15 \\
\hline A2 & Acoustic board 2 & 390 & 15 \\
\hline A3 & Traditional OPF panel & 400 & 20 \\
\hline $1 / 1$ & OPF panel (pattern 1) & 400 & 20 \\
\hline $2 / 1$ & OPF panel (pattern 2) & 400 & 20 \\
\hline $3 / 1$ & OPF panel (pattern 3) & 400 & 20 \\
\hline $4 / 1$ & OPF panel (pattern 4) & 400 & 20 \\
\hline $5 / 1$ & OPF panel (pattern 5) & 400 & 20 \\
\hline $6 / 1$ & OPF panel (pattern 6) & 400 & 20 \\
\hline
\end{tabular}

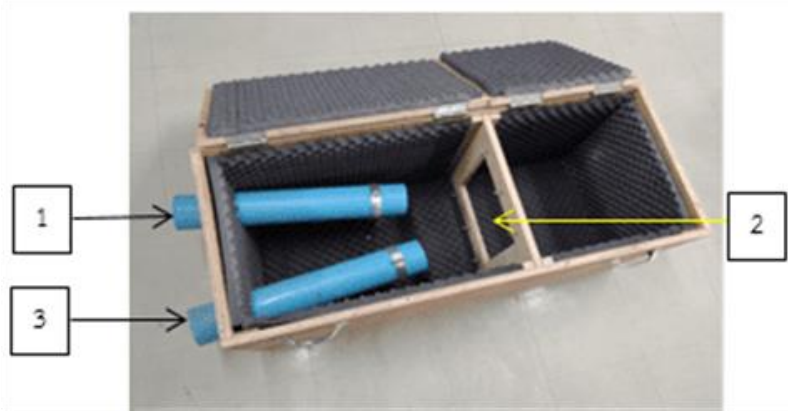

Fig. 11. Tool positions in the sound test box: 1. Position of sound source, Position of samples, 3. Position of sound level meter.

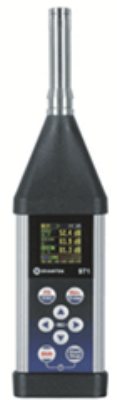




\section{Fig. 12. Sound level meter Svantek, model: SVAN971}

It is noted that the tests were implemented in a soundabsorbing box to decrease the effect of the environmental noise, generally ranging from 10 to $50 \mathrm{dBA}$.

\section{REsults AND Discussion}

Density of the panel samples was determined to be 400 $\mathrm{kg} / \mathrm{m}^{3}$ (panel code $3.1-3.4$ ). It was found that the samples had an average density of $0.36 \mathrm{~g} / \mathrm{cm}$. complying with the standard of Insulating Board (Library of Congress Cataloging - In Publication Data), which requires the density to be ranging from 0.14 to $0.43 \mathrm{~g} / \mathrm{cm}^{3}$. The comparison between the average density of the samples and the required density is shown in Fig. 13.

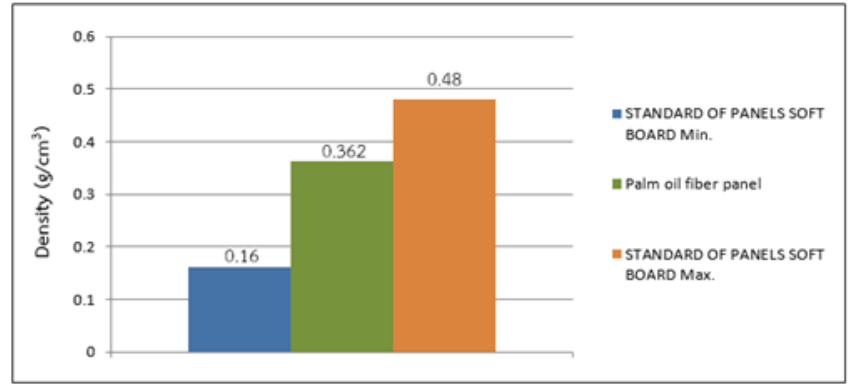

Fig. 13. Fig. 13. Comparison of density

The results from the water absorption test illustrated that an average water absorption of samples was 9.46 percent for sample set number 3 . Furthermore, the water absorption of the samples with the density of $400 \mathrm{~kg} / \mathrm{m} 3$ meets the European standard of soft board, which requires an average water absorption to be ranging from 1 to 10 percent. The comparison between the average water absorption of the samples and the requirement from the standard is shown in Fig. 14

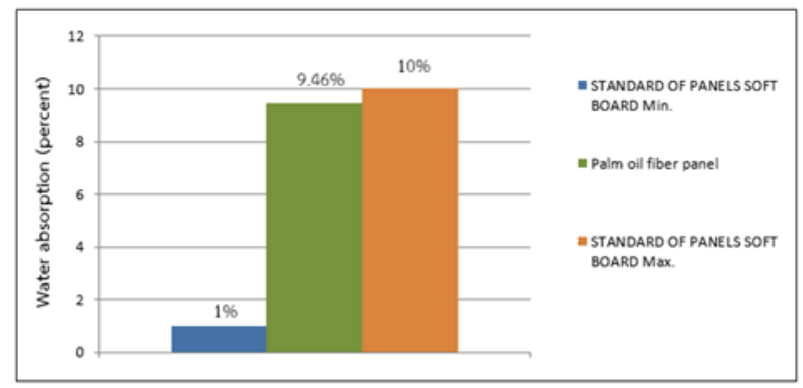

Fig. 14. Comparison of modulus of rupture

The results from the modulus of rupture test indicated that the average modulus of rupture was 5.023 MPa for sample set number 3 , and the density was $0.435 \mathrm{MPa}$. This complies with the standard of Insulating Board (Library of Congress Cataloging - In - Publication Data), which requires the modulus of rupture to be ranging from 1.38 to $5.52 \mathrm{MPa}$. In addition, it was found that an increase in the density resulted in an increase in the modulus of rupture. The comparison between the modulus of rupture of the samples and the required modulus of rupture is shown in Fig. 15.

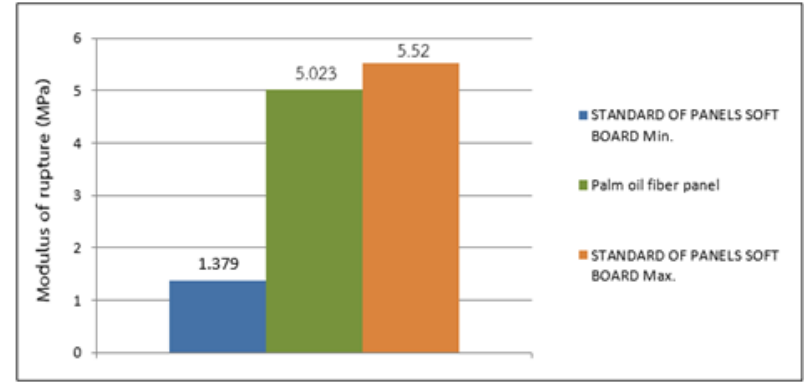

Fig. 15. Comparison of modulus of rupture

The results from the modulus of elasticity test showed that the average modulus of elasticity was $233.18 \mathrm{Mpa}$. For sample set number 4 , and the density was $0.435 \mathrm{MPa}$. This complies with the standard of Insulating Board (Library of Congress Cataloging - In - Publication Data), which requires the modulus of elasticity to be ranging from 1.379 to $5.52 \mathrm{MPa}$. The comparison between the modulus of elasticity of the samples and the required modulus of elasticity is shown in Fig. 16.

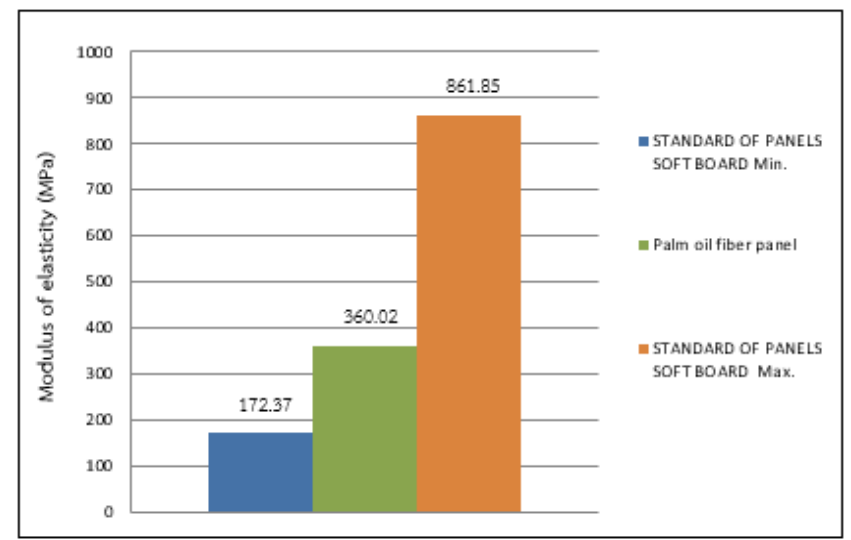

Fig. 16. Comparison of modulus of elasticity

The results from the test of the tensile strength perpendicular to the surface showed that the average tensile strength perpendicular to the surface was $0.3959 \mathrm{Mpa}$ for sample set number 4 , and the density was $0.435 \mathrm{MPa}$. The results exceeded the standard of Strength and Mechanical Properties of Soft Boards (Library of Congress Cataloging - In - Publication Data), which requires the tensile strength perpendicular to the surface to be ranging from 0.16 to $0.4 \mathrm{MPa}$. It implied that oil palm fibers have high cohesion. The comparison between the tensile strength perpendicular to the surface of the samples and those required by the standard is shown in Fig. 17. 


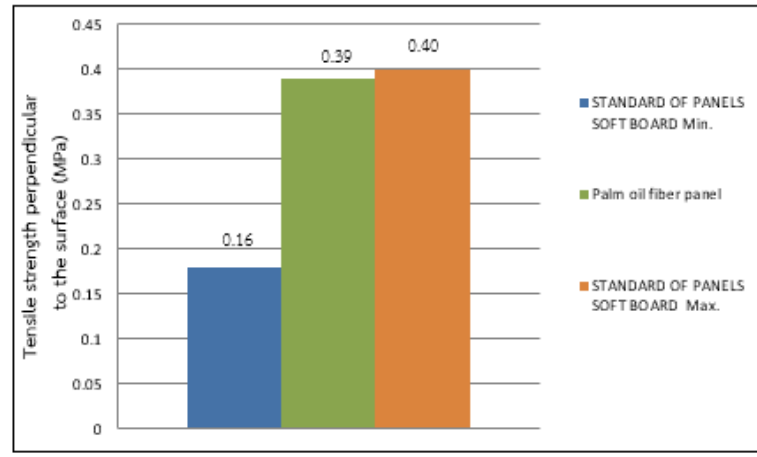

Fig. 17. Comparison of tensile strength perpendicular to the surface

The physical and mechanical properties of the developed OPF panel from the experimental results are similar to those of the traditional OPF panel [3].

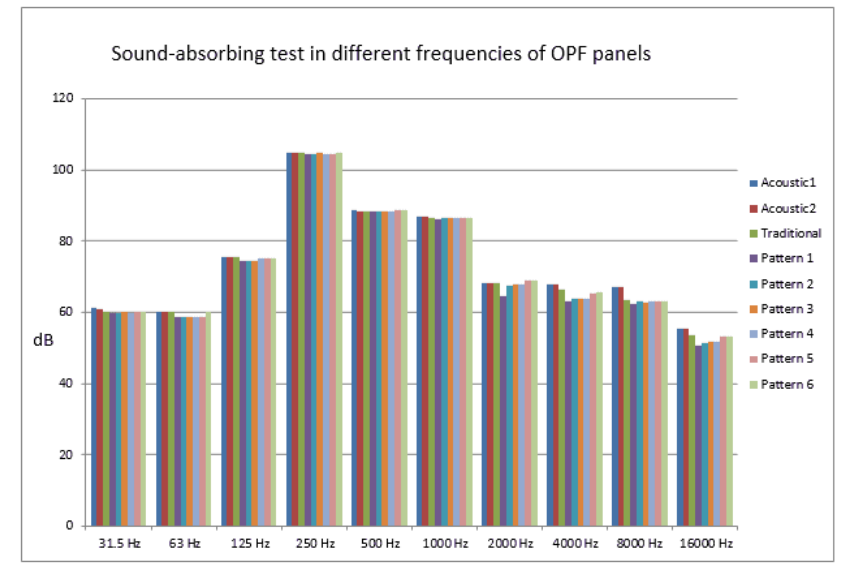

Fig. 18. Sound-absorbing test in different frequencies of OPF panels

The results of sound-absorbing test in different frequencies of OPF panels with the density of $400 \mathrm{~kg} / \mathrm{m} 3$ is illustrated in Fig. 18. It was found that OPF panels with all surface design patterns had an average sound-absorbing and traditional OPF panel had. Panel with pattern 1 performed the greatest average soundabsorbing capacity at frequencies ranging from $63 \mathrm{~Hz}$. to 16,000 $\mathrm{Hz}$. Furthermore, holes on the surfaces of OPF panels affected sound-absorbing capacity. Smaller holes had better soundabsorbing capacity in medium and high frequencies, while larger holes are superior in low frequency. The results corresponds to the sound-absorbing quality of perforated panels which is suitable for the middle-frequency sound [4].

\section{CONCLUSION}

Sound-absorbing property of the developed OPF panels with holed surface are greater than that of the traditional OPF panels and acoustic boards. It was found that different sizes of holes have an effect on sound-absorbing capacity. The developed OPF panels showed a good sound-absorbing capability in mediumto-high frequency $(250 \mathrm{~Hz}-2000 \mathrm{~Hz})$. For the physical and mechanical properties, the OPF panels have the density of 400 $\mathrm{kg} / \mathrm{m} 3$ complying with the standard of strength and mechanical properties of soft boards

\section{RECOMMENDATION}

The research found that the studied OPF panels with the density of $400 \mathrm{~kg} / \mathrm{m}^{3}$ can increase the efficiency of sound absorption by drilling holes on the panel surfaces. In addition, the physical and mechanical properties of the OPF panels meet the standard of Strength and Mechanical Properties of Soft Boards. Thus, it can be developed to be commercial product for sound-absorbing insulation and interior design materials, suitable for meeting room or music room.

Further research can focus on improving the efficiency of sound absorption by forming the surface of the oil palm fiber during hot pressing process

\section{REFERENCES}

[1] W. Oonjittichai, "Wood-alternative materials," Wood Industry Development Group, Forest Research and Development Bureau, Royal Forest Department. Bangkok, p.180, 2012.

[2] V. Desarnaulds, E. Costanzo, A. Carvalho, and B. Arlaud, Sustainability of acoustic materials and acoustic characterization of sustainable materials. In Proceedings of the 12th International Congress on Sound and Vibration, 2005

[3] K. Homaswin, "Sound-absorbing insulation panel from palm oil fiber," In Proceedings of the 9th National Congress of Southern College of Technology on Social Innovation: Innovation for Society, 2019.

[4] P. Rangsirak, Noise control of buildings (instructional materials). Faculty of Architecture, King Mongkut's Institute of Technology Ladkrabang, 1998.

[5] Phichet Durongkawarot, Yuwadee Sansaneeyarat, Guidelines for the development of coconut and coconut products in Thailand. Knowledge Network Institute of Thailand, 2005

[6] R.B. Hoadley, Understanding wood: a craftsman's guide to wood technology. Taunton Press, 2000. 